\title{
Apparatus for collecting voice chronography data
}

\author{
EARL F. BROWN \\ Bell Telephone Laboratories, Holmdel, New Jersey 07733
}

\begin{abstract}
The conversion of speech into on-off pulse patterns representing talk spurts and pauses is a major tool used by psychologists in analyzing interactive communications behavior. We describe a conversion method and a means for interfacing its output with a computer, which allows the collection of large amounts of speech data from one or more sources. The major improvement over prior art is the ability to reject cross talk between neighbor speakers while permitting the detection of simultaneous speech. In the evaluation of the performance of the apparatus, no errors were detected for single-party speaking. Infrequent small errors occurred in the detection and duration of talk spurts and pauses during two- and three-party speaking, but these would probably be acceptable in most applications.
\end{abstract}

Psychologists have been using noncontent speech measures for studying interactive conversational speech patterns for many years. Norwine and Murphy (1938) developed an oscillographic recording technique that is clearly accurate but not convenient. This was followed closely by Chapple's (1939) technique of using a third party to do "on-off" signaling representing talk spurts and pauses, respectively. The analysis of large amounts of speech activity data was made more practical by the development of computer methods by Brady (1965) and Cassotta, Feldstein, and Jaffe (1964). These methods of converting speech to on-off patterns for analysis were, in general, designed for dyadic conversations.

A behavioral study of video conferencing (Brown, Geller, Goodnow, Hoecker, \& Wish, in press; Brown, Limb, \& Prasada, 1978; Hoecker, Brown, Wish, \& Geller, 1978; Mitchell, 1970) required the detection and analysis of speech patterns for up to seven subjects seated on one side of a table conversing with up to seven subjects similarly seated at another location 35 miles away. The large amounts of speech data generated required a computer-interpretable methodology and use of the computer's disk memory. The close proximity of the subjects at each location required a method of preventing cross talk while allowing the detection of simultaneous speech patterns of two or more subjects.

To meet these requirements, an audio-to-data converter was built that converts speech of up to seven subjects at one location into computer-interpretable on-off pulse patterns representing, respectively, talk spurts and pauses. The converter is designed to detect

D. G. Hoecker suggested this project. P. D. Bricker's contributions in evaluating the performance of the converter were invaluable. J. D. Robbins wrote the computer programs for receiving and storing the speech data on the computer's disk files. single-party and multiparty speaking and to reject cross talk.

This paper describes the audio-to-data converter and the computer interface and reports on the performance of the audio-to-data converter.

\section{GENERAL DESCRIPTION OF APPARATUS}

A simplified block diagram of the apparatus is shown in Figure 1. The apparatus consists of seven lapel microphones, amplifiers, and cross-talk rejection circuits, plus a computer interface circuit. All of the circuits used off-the-shelf integrated circuits with very few external components.

\section{Computer Interface}

The central part of the computer interface (Figure 2) is a universal asynchronous receiver transmitter (UART) that converts the 7 data bits from a parallel to a serial format and then adds start and stop bits to form a 10-bit word for transmission over telephone lines to the computer. Included in the computer interface are the facilities to $\log$ on the computer, to call the computer program that sorts and stores the data on disk memory, and to terminate the computer program. These instructions to the computer are stored in a programmable read-only memory (PROM). Signals from the computer pass through the UART and detector circuits, causing appropriate light-emitting diodes to glow, indicating to

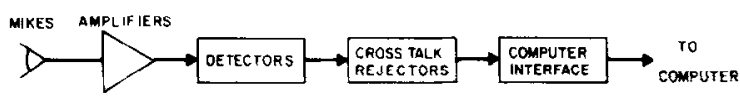

Figure 1. Simplified block diagram of audio-to-data converter and computer interface showing the basic building blocks of the apparatus. 


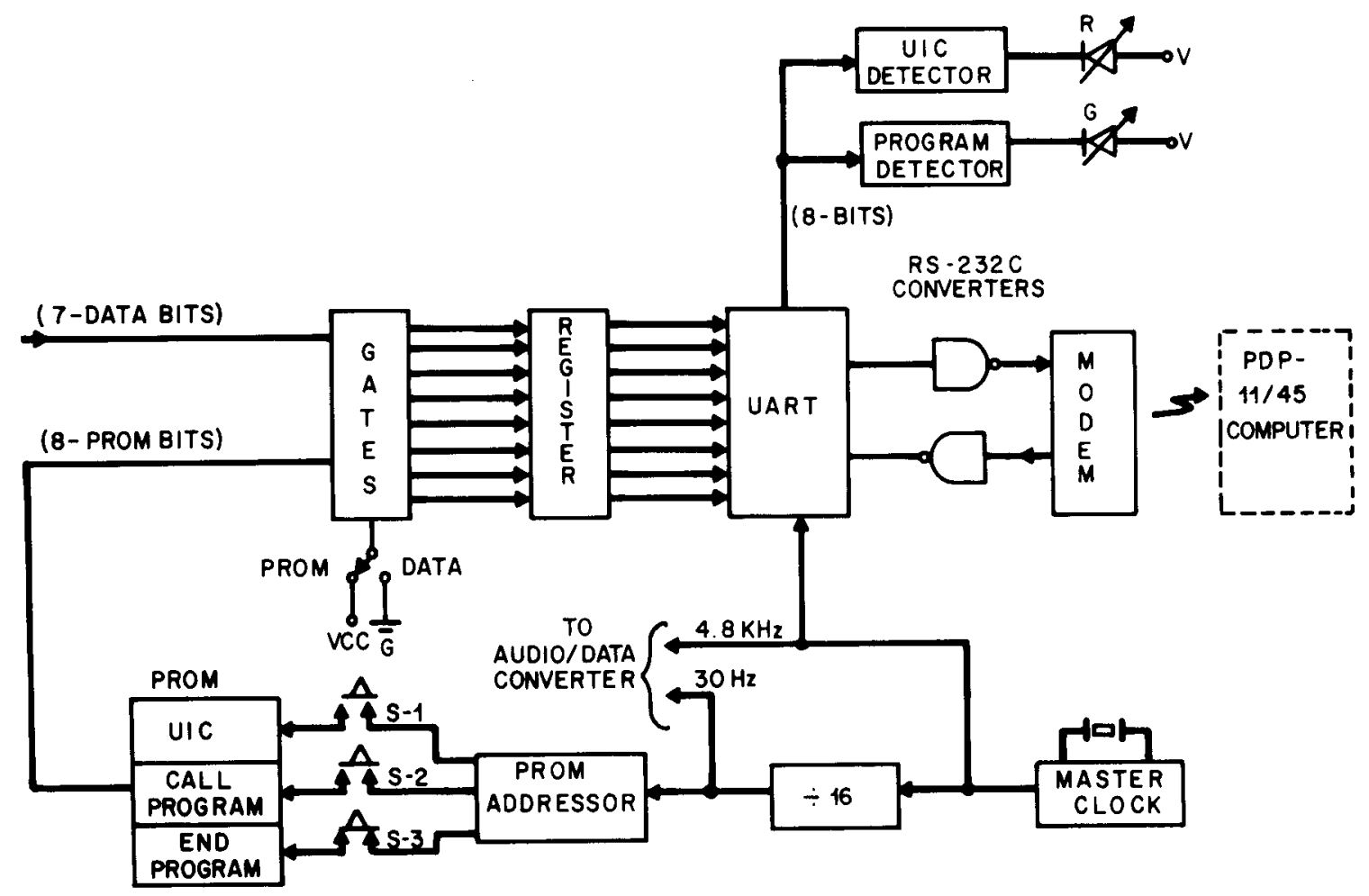

Figure 2. Block diagram of the computer interface. The basic function of this circuit is to convert the on-off speech data from a parallel to a serial format for transmission to the computer. Additional functions allow the experimenter to "log-on" the computer, call program for processing the data, and terminate the program, plus indicating when the experimenter has logged on the computer and when his program is ready to receive data.

the experimenter when he has logged on the computer and when his program is ready to sort the data.

The UART clock conveniently generates a $4.8 \mathrm{kHz}$ and a $30-\mathrm{Hz}$ signal that are used in the audio-to-data converter.

\section{Audio-to-Data Converter}

The audio-to-data converter (Figure 3 ) is designed to convert speech signals into on-off signals and to prevent a speaker from generating false pulses in his neighbor's channel while allowing the generation of on-off pulses in their respective channels when more than one speaker is talking.

The output of each Electret lapel microphone is amplified $50 \mathrm{~dB}$ and applied to a differential comparator. When the instantaneous speech signal exceeds a threshold, the comparator produces an output level until the speech signal falls below that threshold. The comparator's output is sampled at a $4.8 \mathrm{kHz}$ rate and applied to a counter ${ }^{1}$ and then to a majority logic circuit (MLC). The MLC gates are interrogated at a $30-\mathrm{Hz}$ rate $(10 \%$ the baud rate). The counters and MLCs are reset by the $30-\mathrm{Hz}$ pulse.

Rejection of adjacent neighbor's cross talk is accomplished as follows: Assume that Microphone 2 is active and the speaker's voice is also being picked up by Microphones 1 and 3 at a lower level. The duration of the pulse out of Comparator 2 will be greater than the duration of the pulses out of Comparators 1 and 3 . Thus the number of samples counted for Channel 2 will be greater and the MLCs will inhibit outputs from Channels 1 and 3 and pass output pulses for Channel 2 .

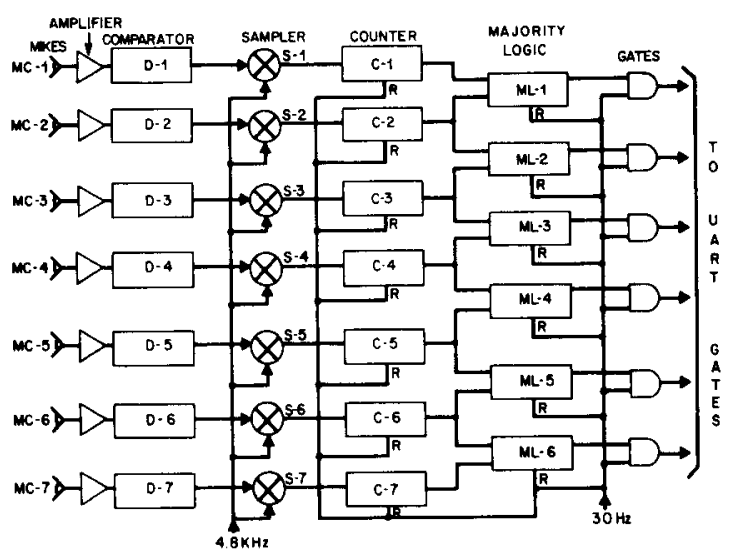

Figure 3. Block diagram of the audio-to-data converter. This circuit compares the audio signal with a reference and produces a pulse as long as the audio signal exceeds the reference, samples these pulses, counts the samples, compares the number of samples in adjacent channels, and passes a signal to the computer interface for the channel with the largest count. 
When neighbors are speaking at the same time, both counters will contain a positive count at each successive $1 / 30-\mathrm{sec}$ sampling point. It can be expected that the larger count will be found on one or the other in a random sequence. Thus the MLC will credit each speaker with some ones and zeros during the simultaneous talking interval. Computer processing later interprets such sequences to fill in the missing pulses using the throw-away and fill-in rules described in this paper.

\section{OBJECTIVES OF PERFORMANCE EXPERIMENTS}

The objective of these experiments was to evaluate the performance of the audio-to-data converter in the detection of talk spurts and pauses and their durations during single, double, and triple talking in three-party conversations. Three experiments were conducted: (1) In Experiment 1 one person spoke into three appropriately placed microphones with the MLC successively inactivated and activated to demonstrate the effectiveness of the MLC circuits in inhibiting cross talk. (2) In Experiment 2 a three-party conversation was simulated by three people reading scripts under seven test conditions in order to test the converter equally under all conditions of single, double, and triple talking. (3) In Experiment 3 two sets of taped three-party conversations were used to measure the performance of the equipment in "real" situations.

\section{DESIGN OF EXPERIMENTS}

In each of the experiments the on-off pulse patterns produced by the converter were recorded on three tracks of a four-track oscillograph. In interpreting the oscillograph patterns, isolated pulses $<66 \mathrm{msec}$ (two pulses) are thrown away (ignored) and gaps $\leqslant 299 \mathrm{msec}$ (equivalent to nine pulse periods) are filled in (bridged). The resulting on-off patterns are defined as talk spurts and pauses (Brady, 1965).

In each of these experiments the threshold of the audioto-data converter was set at $40 \mathrm{dBm}$.

\section{Experiment 1}

In this experiment three Electret lapel microphones were used. The two "far" microphones were placed $8 \mathrm{~cm}$ above a tabletop and $36 \mathrm{~cm}$ in front of the speaker. The "near" (center) microphone was hand held about $8 \mathrm{~cm}$ in front of the speaker's mouth. The outputs of the microphones were applied to the audio-to-data converter and then to the oscillograph.

\section{Experiment 2 (Simulated Conversation)}

Three subjects were seated on one side of a table $75 \mathrm{~cm}$ apart in a $3.7 \times 7.3 \times 3.0 \mathrm{~m}$ room with sheet metal walls and concrete floors covered with vinyl tile. Each subject wore an Electret lapel microphone. Each subject was assigned a script, which he read for the tests he participated in. Each script contained about 90 words and took about $30 \mathrm{sec}$ to read. The speaking order for the seven tests is shown in Table 1.

The on-off pulses from the converter were recorded on three tracks of an oscillograph. The audio waveforms were similarly recorded and used as a reference to measure the accuracy of performance of the audio-to-data converter. The threshold for speech in the audio waveforms was determined as follows:
Table 1

\begin{tabular}{cccc} 
Test & Speakers & Test & Speakers \\
\hline 1 & 1 (Left Side) & 4 & 1 and 2 \\
2 & 2 (Center) & 5 & 2 and 3 \\
3 & 3 (Right Side) & 6 & 1 and 3 \\
& & 7 & 1,2 , and 3 \\
\hline
\end{tabular}

The author listened to the audio to detect known quiet times in the audio waveforms. The magnitude of the audio waveform during quiet times provided a measure of system noise. Signals perceptually detected to be above the noise level were interpreted as speech.

\section{Experiment 3 (Three-Party Conversations)}

About $5 \mathrm{~min}$ each of two three-party conversations recorded during the video conferencing experiment were used in this experiment.

The recorded audio was reproduced on three $20-\mathrm{cm}$ speakers in the same room used in Experiment 2. The loudspeakers were placed on a table top $75 \mathrm{~cm}$ apart with a cardiod microphone placed $10 \mathrm{~cm}$ in front of each loudspeaker. The output of the microphones was applied to the audio-to-digital converter, and its output was recorded on the oscillograph. Then the audio waveforms to be used as a reference were recorded on the oscillograph.

\section{RESULTS}

\section{Experiment 1}

Inspection of Figure 4 demonstrates the ability of the MLC to reject cross talk between adjacent microphones. In Figure 4A, in which the MLC is inactive, 14 erroneous talk spurts are generated by the far microphones using the throw-away and fill-in times described earlier; in Figure 4B, where the MLC is active, only two isolated "false" pulses are generated.

\section{Experiment 2 (Simulated Conversation)}

In this experiment the throw-away and fill-in times defined earlier were used to identify talk spurts and pauses. Then a scale marked off in $100-\mathrm{msec}$ increments was used to measure the durations of talk spurts and pauses in the two sets of oscillograms. The talk-spurt and pause measurements were compared using the audio waveforms as a reference to determine the accuracy of performance of the audio-to-data converter.

There were 99 talk spurts and 87 pauses, for a total of 176 events. No errors were generated for Tests 1 , 2 , and 3 when only one person was speaking, nor during Test 6 when the two distant subjects were speaking. Otherwise, errors occurred in two forms. One was a time error occurring when the talk spurt or pause time began too early or too late. This in effect caused two errors; that is, an error on the first event caused an equal timing error on the next event. There were eight of these double errors during two-party talking in Tests 4 and 5 and four during three-party talking. The average error was $.15 \mathrm{sec}$, with a minimum error of $.1 \mathrm{sec}$ and a maximum error of $.2 \mathrm{sec}$. The second type of error was 


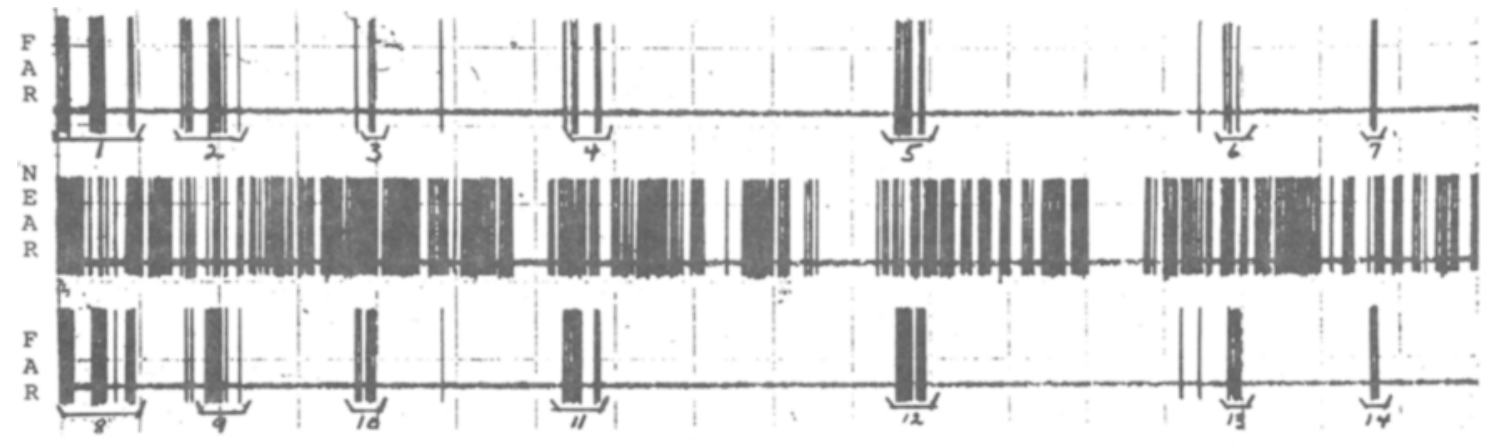

FIGURE 4A

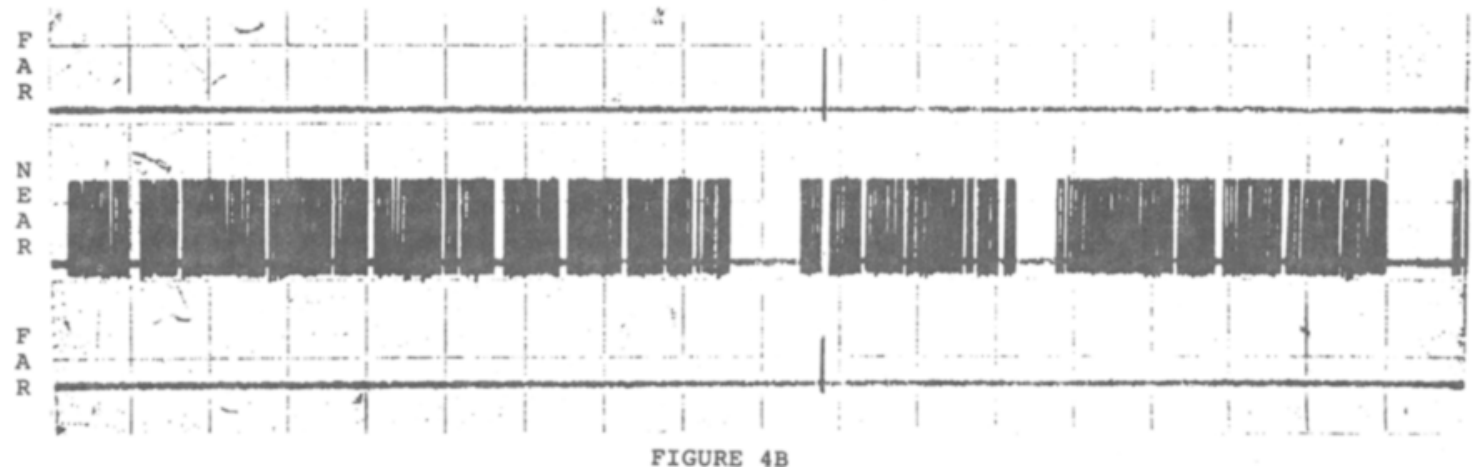

Figure 4. Oscillographic recordings of on-off speech patterns of the experimenter with his microphone held $8 \mathrm{~cm}$ in front of himself and with the two "far" microphones placed $36 \mathrm{~cm}$ in front of him: (a) with cross-talk rejection circuit deactivated, (b) with cross-talk rejection circuit activated. (The pulses were "inked over" to increase contrast.) Note in Figure 4A that 14 erroneous talk spurts are generated, whereas in Figure 4B only two isolated false pulses are generated.

the detection of false pause events. This was caused by the fact that two adjacent channels cannot generate pulses during the same period. Thus, when one speaker talks much louder than his neighbor, an erroneous pause is generated in the neighbor's on-off pulse pattern. No false pauses were generated during Tests 1, 2, 3, and 6 . Five false pauses were generated during two-party talking (Tests 4 and 5), and three during three-party talking. Seven of these false pauses had durations of $.3 \mathrm{sec}$; one was $.4 \mathrm{sec}$. If the fill-in time had been increased to $.399 \mathrm{sec}$, only one false event would have occurred.

\section{Experiment 3 (Three-Party Conversations)}

The same throw-away and fill-in times and measurement methods used in Experiment 2 were used in this experiment.

There were 199 talk spurts and 199 pauses during these two sessions. There were 38 cases of double talking and 8 cases of triple talking. Double talking occurred for $29.0 \mathrm{sec}$, with an average duration of $.76 \mathrm{sec} / \mathrm{case}$. Triple talking occurred for $8.2 \mathrm{sec}$, with an average duration $1.02 \mathrm{sec} / \mathrm{case}$. Double or triple talking occurred about $6.2 \%$ of the total conversation time.
No errors were detected during single-party talking. There were 10 timing errors and one event error during two- and three-party talking. Six timing errors occurred during two-party talking and four during three-party talking. The average timing error was $.16 \mathrm{sec}$, with a minimum of $.1 \mathrm{sec}$ and a maximum of $.4 \mathrm{sec}$. The event error that occurred during three-party talking caused a false .6-sec pause error. Therefore, out of 199 talk spurts, 188 were measured the same $( \pm .1 \mathrm{sec})$ by the audio-to-data converter as by visual inspection of the acoustic oscillograms.

\section{DISCUSSION}

The audio-to-data converter works well in the detection and measurement of talk-spurt and pause durations for single-party talking. During multiparty talking, small timing errors in the duration of talk spurts and pauses occur infrequently. Event errors occur with even less frequency. During two three-party conversations lasting about $5 \mathrm{~min}$ each, 199 talk spurts occurred, of which 188 were correct $( \pm .1 \mathrm{sec})$. Ten timing errors were generated, with an average error of $.16 \mathrm{sec}$, and one event error caused a false .6 -sec pause error. Errors 
of this order and magnitude are probably less than those due to fluctuations in speech level, "trailing-off" of speech and noise, and should not significantly effect the overall results.

The most significant improvement of this method over prior art is its ability to minimize the effect of cross talk in the detection and measurement of talk spurts and pauses; with given postprocessing and stated fill-in times, it permits the detection of simultaneous speech when several speakers are seated in close proximity. This is demonstrated by Experiment 1, which shows that the MLC does defeat cross talk; Experiment 2, which shows that the MLC registers deliberately maximized simultaneous talking; and Experiment 3 , which uses more natural speech material and renders it with usable accuracy.

\section{REFERENCES}

Brady, P. T., A technique for investigating on-off patterns of speech. Bell System Technical Journal, 1965, 44, 1-22.

Brown, E. F., Geller, V. J., Goodnow, III, J. E., Hoecker, D. G., \& Wish, M. Some objective and subjective differences between communication over two videoconferencing systems. IEEE Transactions on Communications, in press.

Brown, E. F., Limb, J. O., \& Prasada, B. A continuous presence video conferencing system. In Conference record,
1978 National Telecommunications Conference (Vol. 3), 1978.

Cassotta, L., Feldstein, S., \& Jaffe, J., AVTA: A device for automatic vocal transaction analysis. Journal of the Experimental Analysis on Behavior, 1964, 7, 99-104.

CHAPPLE, E. D., Quantitative analysis of the interaction of individuals, Proceedings of the National Academy of Science, 1939, 25, 58-67.

Hoecker, D. G., Brown, E. F., Wish, M., \& Geller, V. J. A behavioral comparison of communication using voice-switched and continuous presence video conferencing arrangements. In Conference record, 1978 National Telecommunications Conference (Vol. 3), 1978.

Mitchell, D. Better video conferences. Bell Laboratories Record, January 1970 , p. 9.

Norwine, A. C. \& Murphy, J. O. Characteristic time intervals in telephone conversation. Bell System Technical Journal, 1938, 281 .

\section{NOTE}

1. The counter should normally count up to a maximum of 160 pulses $(4,800 / 30)$. To reduce the number of chips and to make the circuit applicable to any sampling and reset rate, the maximum count was set at 15 (one chip). When a maximum count of 15 is reached, that counter and the two neighbor counters are reset and restarted within a gating period.

(Received for publication October 12, 1979 accepted November 1,1979 ) 